\title{
Experimental Study on Neutron Correlation Analysis for a Subcritical System Driven by a Pulsed Spallation Neutron Source in KUCA
}

\author{
Kunihiro Nakajima ${ }^{1}$, Kazuki Takahashi ${ }^{1}$, Atsushi Sakon ${ }^{2}$, Sin-ya Hohara ${ }^{2}$, \\ Tadafumi Sano ${ }^{2}$, Masao Yamanaka ${ }^{3}$, Cheol Ho Pyeon ${ }^{3}$, and Kengo Hashimoto \\ ${ }^{1}$ Graduate School of Science and Engineering, Kindai University \\ 3-4-1 Kowakae, Higashi-Osaka, Osaka, 577-8502, Japan \\ ${ }^{2}$ Atomic Energy Research Institute, Kindai University \\ 3-4-1 Kowakae, Higashi-Osaka, Osaka, 577-8502, Japan \\ ${ }^{3}$ Institute for Integrated Radiation and Nuclear Science, Kyoto University \\ Asashiro-nishi, Kumatori-cho, Osaka, 590-0494, Japan
}

kunihiro_nakajima@nk-mail.com, takahashi.kazuki0524@gmail.com,sakon@kindai.ac.jp, hohara@kindai.ac.jp,t-sano@kindai.ac.jp,m-yamanaka@rri.kyoto-u.ac.jp, pyeon@rri.kyoto-u.ac.jp, kengoh@pp.iij4u.or.jp

\begin{abstract}
The Feynman- $\alpha$ and the Rossi- $\alpha$ methods have been frequently employed to determine the subcriticality of subcritical reactor systems driven by Poisson source such as Am-Be neutron source. In actual accelerator-driven systems (ADS), a spallation device will be applied as an intense neutron source. This device will be probably operated in a pulse mode and it is impossible to apply a conventional analysis method to determine the subcriticality in any ADS. In previous theoretical studies, some advanced formulae of neutron correlation analysis for spallation neutron source have been presented. However, the experimental study has been hardly reported to date. The major objectives of this study are to examine experimentally an applicability of these complicated formulae to a subcritical reactor system driven by an actual pulsed spallation neutron source and to determine the prompt-neutron decay constant $\alpha$ of the system. To achieve these goals, we constructed an ADS core at the Kyoto University Critical Assembly (KUCA). The core was composed of highly-enriched uranium fuel assemblies surrounded by many polyethylene reflector assemblies. We carried out a series of the Feynman- $\alpha$ and the Rossi- $\alpha$ analyses for the system driven by pulsed spallation source. As a result, the prompt-neutron decay constants were experimentally obtained by using a fitting formula. The prompt-neutron decay constants determined by Feynman- $\alpha$ and Rossi- $\alpha$ analyses agrees with each other within a statistical error range of least-squares fitting.
\end{abstract}

KEYWORDS: Neutron Correlation Analysis, Pulsed spallation source, KUCA, ADS

\section{INTRODUCTION}

The Feynman- $\alpha$ and the Rossi- $\alpha$ methods have been frequently employed to determine the subcriticality of subcritical reactor systems driven by Poisson source such as Am-Be neutron source [1,2]. In actual accelerator-driven system (ADS), a spallation device will be applied as an intense neutron source. This 
device will be probably operated in a pulse mode and spallation neutrons have a large multiplicity. Consequently, the neutron source has not only deterministically a pulsed shape but also stochastically a non-Poisson character [3,4]. Then, it is impossible to apply a conventional analysis method to any ADSs. The conventional correlation analysis is applicable to only a steady Poisson source. In previous theoretical studies, some advanced formulae of neutron correlation analysis for pulsed spallation source have been presented [5]. However, the experimental study considering the pulsed non-Poisson source has been hardly reported. The major objectives of this study are to examine experimentally an applicability of these complicated formulae to a subcritical reactor system driven by an actual spallation neutron source and to determine the prompt-neutron decay constant $\alpha$ of the system. To achieve the above goal, a subcritical reactor system was constructed in the Kyoto University Critical Assembly (KUCA) [6] and the system was driven by a spallation neutron source to carry out a series of the Feynman- $\alpha$ and the Rossi- $\alpha$ analyses for the pulsed spallation source. In this paper, the experimental configuration, measurement system and analysis method are described in Section 2. Results of the Feynman- $\alpha$ and the Rossi- $\alpha$ analyses are described in Section 3. Conclusion of this study describes in Section 4.

\section{EXPERIMENT}

The core configuration and neutron detector placement are shown in Figure 1. The core was composed of highly-enriched uranium fuel assemblies (1/8"p60EUEU) surrounded by many polyethylene reflector assemblies. The system had a lead-bismuth ( $\mathrm{Pb}-\mathrm{Bi})$ target, to which $100 \mathrm{MeV}$ proton beams were injected, to generate spallation reactions [7]. In this experiment, the pulsed proton beams from an accelerator were generated at $30 \mathrm{~Hz}$ repetition rate (repetition period of 1/30 sec). KUCA has six control rods, where three are assigned as safety rods for reactor scram and are referred to as S4, S5 and S6, respectively. Others are rods for adjustment of reactivity and are referred to as $\mathrm{C} 1, \mathrm{C} 2$ and $\mathrm{C} 3$, respectively. In this study, the subcriticality was adjusted by changing the axial positions of these safety and control rods. Center core position was also used for adjusting the subcriticality. We prepared a measurement system as shown in Figure 2. $\mathrm{A} \mathrm{BF}_{3}$ proportional counter (1.0 in. dia.) was placed in a location where the counter was not directly exposed by the spallation source. Table I shows several subcritical patterns for the present experiment. The subcriticalities included in this table were experimentally obtained with the combined use of excess reactivity and control rod worth measured by the positive period method and the rod drop method, respectively. At each subcritical state, time-sequence neutron counts data from the $\mathrm{BF}_{3}$ counter were acquired for more than $10 \mathrm{~min}$. For comparison, we acquired similarly time-sequence data at each system driven by an Am-Be neutron source and an inherent neutron source. Based on these time-sequence data acquired, we carried out the Feynman- $\alpha$ and the Rossi- $\alpha$.

The Feynman- $\alpha$ method is utilizing a deviation of neutron counts accumulated during a gate time from the Poisson distribution. The prompt neutron decay constant can be determined by observing the gate time dependence of the variance-to-mean ratio. In this measurement, time sequence data is recorded in the time stamp format, so it is converted to time sequence data of count rate per $1 \mathrm{msec}$ gate time width. Then a moving-bunching technique [3] was applied to synthesize the counts data within wider gate widths. In this analysis, the variance-to-mean ratios of these counts data were calculated to obtain a gate-width dependence of the ratios.

The Rossi- $\alpha$ method directly observe the decay schema of fission chain. The prompt neutron decay constant can be determined from a conditional probability that, given a neutron count at a time, there is a subsequent count in $\Delta \tau$ about time $\tau$ latter. 

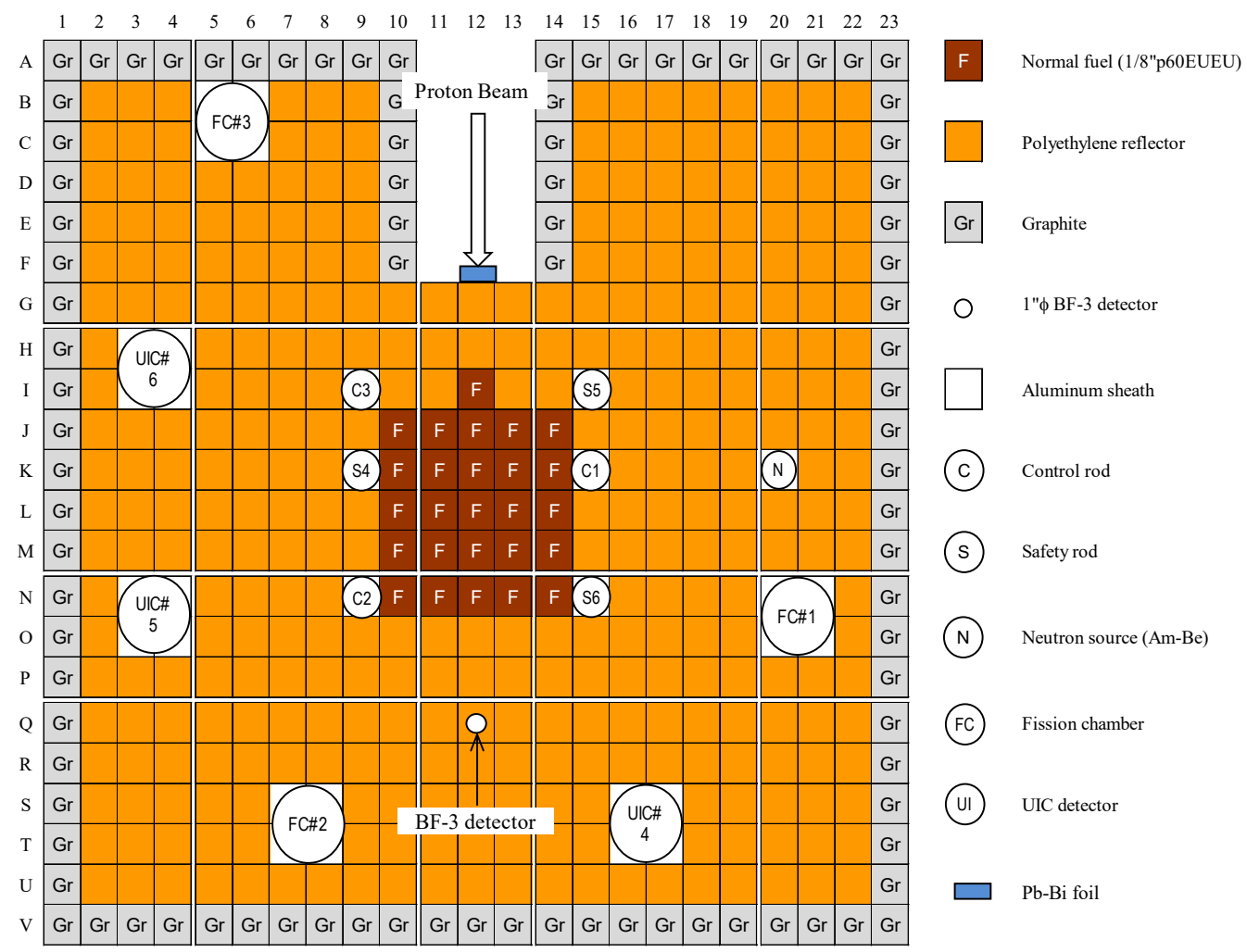

Figure 1. Reactor configuration.

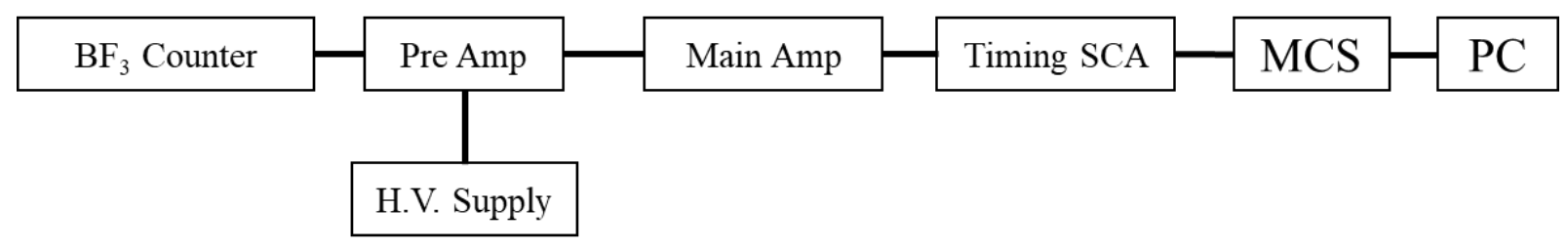

Figure 2. Measurement system.

Table I. Subcritical Pattern

\begin{tabular}{|c|c|c|c|c|c|c|c|c|}
\hline Subcritical & Subcriticality & Center Core & \multicolumn{5}{|c|}{ Control Rod and Safety Rod Position } \\
\cline { 4 - 8 } & $\begin{array}{c}\text { Pattern } \\
{[\% \Delta \mathrm{k} / \mathrm{k}]}\end{array}$ & Position & C1 & C2 & C3 & S4 & S5 & S6 \\
\hline A & 0.347 & U.L. & U.L. & L.L. & U.L. & U.L. & U.L. & U.L. \\
\hline B & 0.704 & U.L. & L.L. & U.L. & U.L. & U.L. & U.L. & U.L. \\
\hline C & 1.343 & U.L. & L.L. & L.L. & L.L. & U.L. & U.L. & U.L. \\
\hline D & 1.838 & U.L. & L.L. & L.L. & L.L. & U.L. & U.L. & L.L. \\
\hline E & 2.834 & U.L. & L.L. & L.L. & L.L. & L.L. & L.L. & L.L. \\
\hline F & 13.27 & L.L. & L.L. & L.L. & L.L. & L.L. & L.L. & L.L. \\
\hline
\end{tabular}

L.L.: Lower Limit Position, U.L.: Upper Limit Position 


\section{RESULT AND DISCUSSION}

Figure 3 shows time-sequence neutron count data measured at each subcritical system driven by spallation neutron source. Because of unstable drive of the accelerator, neutron count rate varied with a significant fluctuation of proton beam intensity, as can be seen in pattern A and B.

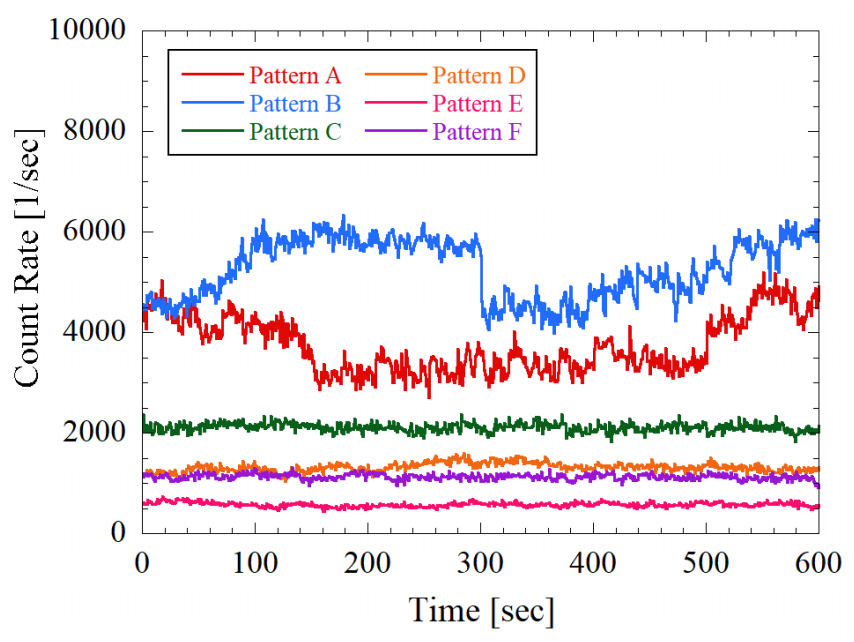

Figure 3. Time variation in neutron count rate.

Figure 4 shows a gate-width dependence of the $Y$ obtained under a respective drives by Am-Be and Inherent neutron source, where the subcritical pattern is " $\mathrm{B}$ " $(-0.704 \% \Delta \mathrm{k} / \mathrm{k})$. The $Y$ is a statistical indication of neutron correlation amplitude and is defined as variance-to-mean ratio minus one of neutron counts within the gate. The prompt-neutron decay constant is determined by least squares fitting of a Feynman- $\alpha$ formula to the $Y$ data. The formula based on a steady Poisson neutron source is shown as follows:

$$
Y(T)=Y_{\infty}\left(1-\left(\frac{1-\exp (-\alpha T)}{\alpha T}\right)\right),
$$

Where, $T$ is a gate time width, $\alpha$ is the prompt-neutron decay constant, $Y_{\infty}$ is a saturated correlation amplitude. The decay constants determined by the Feynman- $\alpha$ analyses under respective drives Am-Be and Inherent source are consistent with each other within fitting error range. Figure 5 shows a gate-width dependence of $Y$ obtained under a drive by pulsed spallation neutron source, where the subcritical pattern is " $\mathrm{B}$ ". The fitting formula based on the spallation neutron source is shown as follows:

$$
\begin{aligned}
Y(T) & =\frac{\lambda_{d} m_{1}}{\alpha}\left[\frac{\exp \left(\alpha\left(T-\frac{[f T]+1}{f}\right)\right)+\exp \left(-\alpha\left(T-\frac{[f T]}{f}\right)\right)+\exp \left(-\alpha\left(T+\frac{1}{f}\right)\right)-2 e^{-\alpha / f}-e^{-\alpha T}}{\alpha T\left(1-e^{-\alpha / f}\right)}\right] \\
& -\frac{\lambda_{d} m_{1}}{\alpha}\left[f T-2[f T]+\frac{[f T]([f T]+1)}{f T}\right]+\frac{\lambda_{d}\left(m_{2}+2 m_{1} Y_{1}\right)}{m_{1} \alpha}\left(1-\frac{1-\exp (-\alpha T)}{\alpha T}\right)+C_{1} T,
\end{aligned}
$$


where $f$ is repletion rate of the pulsed proton beams. $[f T]$ is largest integer less than or equal to $f T$, that is, nearest integer rounded down from $f T$. $\lambda_{d}$ is a probability per unit time of detecting one neutron. $Y_{1}$ is second moment of total number of neutrons generated in fission. $m_{1}, m_{2}$ are the first and second factorial moment of multiplicity distribution of neutrons produced by the spallation reactions per proton bunch. $C_{1} T$ is a correction term considering the beam intensity drifts over a comparable time scale with delayed neutrons. The above formula was applied to the Feynman- $\alpha$ analysis. As a result, the fitting of the improved theoretical formula to the Y data was successful, and the prompt neutron decay constant could be determined. The uncorrelated component originated from the pulsed neutron source is also well expressed.

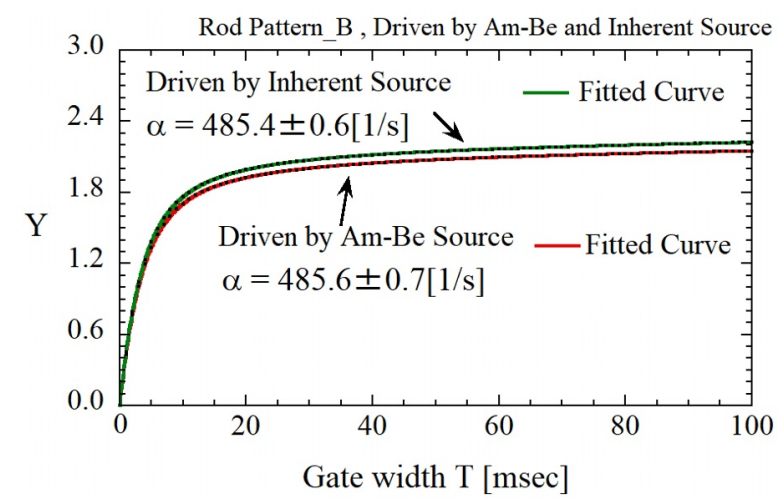

Figure 4. Result of Feynman- $\alpha$ analysis (Am-Be and Inherent Source).

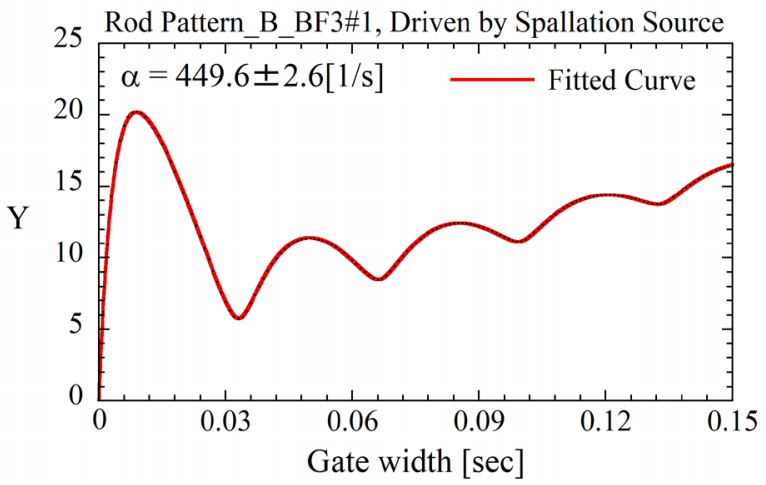

Figure 5. Result of Feynman- $\alpha$ analysis. (Spallation Source).

Figure 6 shows a time-interval dependence of the conditional probability obtained under each drive by $\mathrm{Am}-\mathrm{Be}$ and Inherent neutron source, where the subcritical pattern is " $\mathrm{B}$ ". The prompt neutron decay constant is determined by least-squares fitting of a Rossi- $\alpha$ formula. The fitting formula based on a steady Poisson neutron source is shown as follows:

$$
P(\tau)=D_{1} \exp (-\alpha \tau)+D_{2}
$$

Where $\tau$ is the time interval between two neutron counts, $D_{1}$ is a signal (correlated) constant, $D_{2}$ is another noise (uncorrelated) constant. The above formula was applied to the Rossi- $\alpha$ analysis to determine the prompt neutron decay constants. The decay constants determined by Rossi- $\alpha$ analyses under respective drives by Am-Be and Inherent source agree with each other within fitting error range. The strength of the $\mathrm{Am}-\mathrm{Be}$ neutron source is larger than that of the inherent source and consequently the result of Am-Be source has a larger bias component $\left(D_{2}\right)$ than that of Inherent source.

Figure 7 shows a time-interval dependence of the conditional probability obtained under a drive by the pulsed spallation neutron source, where the subcritical pattern is " $\mathrm{B}$ ". The fitting formula based on the spallation neutron source is shown as follows:

$$
P(\tau)=\frac{f \lambda^{2} d}{2 \alpha}\left[\frac{m_{1}{ }^{2}}{1-e^{-\alpha / f}}\left\{e^{-\alpha / f} e^{\alpha(\tau-[f \tau])}+e^{-\alpha(\tau-[f \tau] / f)}+\left(m_{2}-m_{1}{ }^{2}+2 m_{1} Y_{1}\right) e^{-\alpha \tau}\right\}\right]+C_{2},
$$

where $C_{2}$ is a correction term considering a contribution of delayed neutrons to the conditional probability. The above formula was applied to the Rossi- $\alpha$ analysis. As a result, the fitting of the improved formula is also successful, and the prompt neutron decay constant could be determined. 


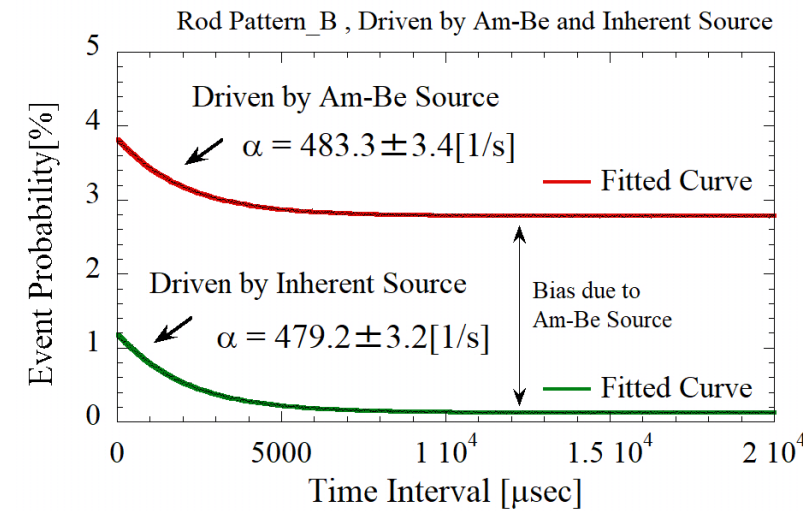

Figure 6. Result of Rossi- $\alpha$ analysis (Am-Be and Inherent Source).

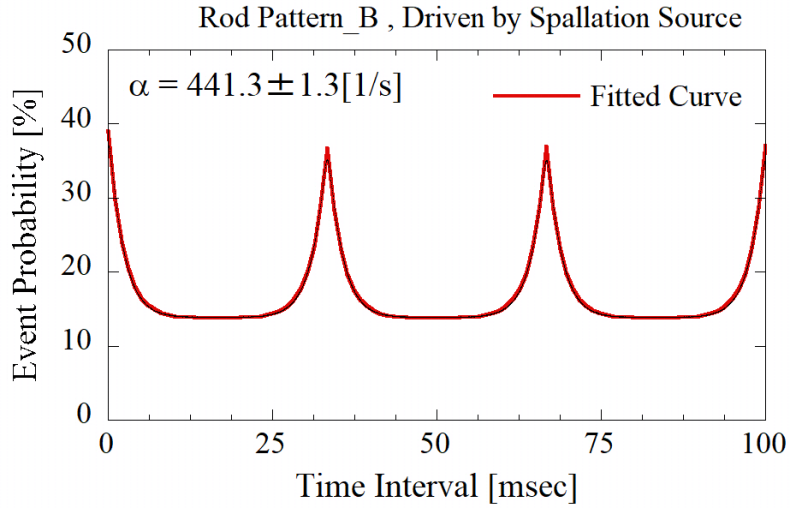

Figure 7. Result of Rossi- $\alpha$ analysis. (Spallation Source).

Similarly, the Feynman- $\alpha$ and the Rossi- $\alpha$ analyses were performed at several subcritical states and the prompt neutron decay constants determined are shown in Table II and Table III, respectively.

Figure 8 shows a comparison of the prompt-neutron decay constants determined from the Feynman- $\alpha$ and Rossi- $\alpha$ analyses under respective drives by the Am-Be, the inherent and the spallation sources. Comparison of the decay constants between Feynman- $\alpha$ and Rossi- $\alpha$ methods agrees with each other within fitting error range. However, in case of the spallation source, the decay constant is different from those obtained under respective drives by the Am-Be neutron source and inherent source. The decay constants under a drive by the spallation source were underestimated compared with those under the other drives.

Table II. Prompt decay constants determined by Feynman- $\alpha$ analysis

\begin{tabular}{|c|r|r|r|}
\hline \multirow{2}{*}{$\begin{array}{c}\text { Subcritical } \\
\text { Pattern }\end{array}$} & \multicolumn{4}{|c|}{ Prompt Neutron Decay Constant $\alpha[1 / \mathrm{sec}]( \pm 2 \sigma)$} \\
\cline { 2 - 5 } & Am-Be Source & \multicolumn{1}{|c|}{ Inherent Source } & Spallation Source \\
\hline A & $370.3 \pm 2.0$ & $386.1 \pm 2.0$ & $353.7 \pm 5.0$ \\
B & $485.6 \pm 1.4$ & $485.4 \pm 1.2$ & $449.6 \pm 2.6$ \\
C & $734.3 \pm 3.8$ & $719.0 \pm 5.2$ & $649.1 \pm 3.6$ \\
D & $893.5 \pm 8.2$ & $907.2 \pm 5.4$ & $858.4 \pm 4.4$ \\
E & $1385.6 \pm 22.4$ & $1257.6 \pm 21.2$ & $1194.7 \pm 10.6$ \\
F & $3010 \pm 280$ & $2693 \pm 160$ & $2225.5 \pm 13.6$ \\
\hline
\end{tabular}


Table III. Prompt decay constants determined by Rossi- $\alpha$ analysis

\begin{tabular}{|c|r|r|c|}
\hline \multirow{2}{*}{$\begin{array}{c}\text { Subcritical } \\
\text { Pattern }\end{array}$} & \multicolumn{3}{|c|}{ Prompt Neutron Decay Constant $\alpha[1 / \mathrm{sec}]( \pm 2 \sigma)$} \\
\cline { 2 - 5 } & Am-Be Source & \multicolumn{1}{|c|}{ Inherent Source } & Spallation Source \\
\hline A & $374.3 \pm 2.0$ & $373.5 \pm 2.6$ & $353.6 \pm 1.0$ \\
B & $486.7 \pm 3.4$ & $481.8 \pm 3.2$ & $441.3 \pm 1.4$ \\
C & $728.5 \pm 8.2$ & $727.3 \pm 10.8$ & $658.6 \pm 2.8$ \\
D & $925.9 \pm 14.8$ & $938.1 \pm 13.0$ & $813.5 \pm 3.6$ \\
E & $1309.4 \pm 33.2$ & $1256.9 \pm 34.4$ & $1098.9 \pm 6.2$ \\
F & $2675 \pm 230$ & $2877 \pm 50$ & $2028.9 \pm 12.6$ \\
\hline
\end{tabular}

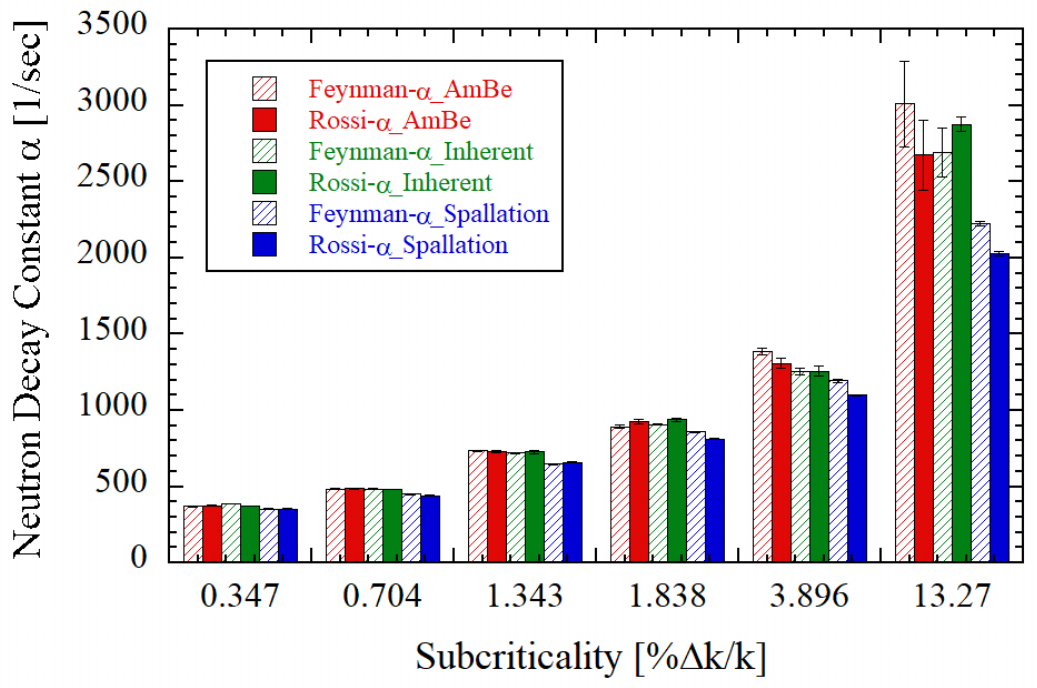

Figure 8. Comparison of the prompt decay constant.

\section{CONCLUSION}

The Feynman- $\alpha$ and the Rossi- $\alpha$ methods have been frequently employed to determine the subcriticality of subcritical reactor systems driven by Poisson source such as Am-Be neutron source. In accelerator-driven system (ADS), a spallation device will be applied as an intense neutron source. This device will be probably operated in a pulse mode and it is impossible to apply a conventional analysis method to these pulsed nonPoisson source of ADSs. In previous theoretical studies, some advanced formulae of neutron correlation analysis for pulsed spallation neutron source have been presented. However, the experimental study has been hardly reported to date. The major objectives of this study are to examine experimentally an applicability of these complicated formulae to a subcritical reactor system driven by the pulsed spallation neutron source and to determine the prompt-neutron decay constant $\alpha$ of the system. To achieve these goals, the Feynman- $\alpha$ and the Rossi- $\alpha$ analyses for a pulsed spallation source has been performed in several subcritical systems at the KUCA. As a result, the prompt-neutron decay constants were experimentally obtained by using a fitting formula. The respective decay constants obtained from the Feynman- $\alpha$ and the 
Rossi- $\alpha$ analyses agrees with each other within fitting error range. However, in case of the spallation source, the decay constants were underestimated compared with those under the other drives.

\section{ACKNOWLEDGMENTS}

The present work was performed as a joint research program of the KUCA at the Institute for Integrated Radiation and Nuclear Science, Kyoto University.

\section{REFERENCES}

1. R. P. Feynman, F. De Hoffmann, R. Serber, "Dispersion of the neutron emission in U-235 fission," J. Nucl. Energy, 3, pp.64-69 (1956).

2. J.D.Orndorff, "Prompt Neutron Periods of Metal Critical Assemblies", Nucl.Sci.Eng., Vol.2, pp.450-460 (1957).

3. R. Okuda, et al., "An improved Feynman- $\alpha$ analysis with a moving-bunching technique," $J$. Nucl. Sci. Technol., 53, pp.1647-1652 (2016).

4. H. Taninaka, et al., "Feynman- $\alpha$ analysis for a thermal subcritical reactor system driven by an unstable 14MeV neutron source," J. Nucl. Sci. Technol., 48, pp.1272-1280 (2011).

5. S.B. Degweker, "Reactor Noise in Accelerator Driven Systems", Ann .Nucl. Energy, 30, pp.223243 (2003).

6. T. Misawa, H. Unesaki, C. H. Pyeon, "Nuclear Reactor Physics Experiment", Kyoto University Press, Japan (2010).

7. C. H. Pyeon, et al., "Neutron characteristics of Solid targets in accelerator-driven system with 100 MeV protons at Kyoto University Critical Assembly," Nucl. Technol., 192, pp.181-190 (2015). 